\title{
RESULTS ON DOMINATION NUMBER AND BONDAGE NUMBER FOR SOME FAMILIES OF GRAPHS
}

\author{
G. Hemalatha ${ }^{1}$, P. Jeyanthi ${ }^{2} \S$ \\ ${ }^{1}$ Department of Mathematics \\ Shri Andal Alagar College of Engineering \\ Mamandur, Kancheepuram, Tamil Nadu, INDIA \\ ${ }^{2}$ Research Centre, Department of Mathematics \\ Govindammal Aditanar College for Women \\ Tiruchendur 628 215, Tamil Nadu, INDIA
}

\begin{abstract}
Let $G=(V, E)$ be a simple graph on the vertex set $V$. In a graph $G$, A set $S \subseteq V$ is a dominating set of $G$ if every vertex in $V S$ is adjacent to some vertex in $S$. The domination number of a graph $G \gamma(G)$ ] is the minimum size of a dominating set of vertices in $G$. The bondage number of a graph $G[B d \gamma(G)]$ is the cardinality of a smallest set of edges whose removal results in a graph with domination number larger than that of $G$. In this paper we establish domination number and the bondage number for some families of graphs.
\end{abstract}

AMS Subject Classification: $05 \mathrm{C} 69$

Key Words: dominating set, bondage number, domination number, cocktail party graph, coxeter graph, crown graph, cubic symmetric graph, doyle graph, folkman graph, levi graph, icosahedral graph

\section{Introduction}

The domination in graphs is one of the major areas in graph theory which attracts many researchers because it has the potential to solve many real life problems involving design and analysis of communication network, social net-

Received: October 17, 2015

Published: April 19, 2016

${ }^{\S}$ Correspondence author (c) 2016 Academic Publications, Ltd.

url: www.acadpubl.eu 
work as well as defence surveillance. Many variants of domination models are available in the existing literature. The literature on domination and related parameters has been surveyed and beautifully presented in the two books by Haynes, Hedetniemi and Slater [5, 6]. The domination number of a graph $G[\gamma(G)]$ is the minimum size of a dominating set of vertices in $G$. Considering possible link faults in the real world, Fink et al.[4] introduced the concept of the bondage number. However, the first result on bondage numbers was proposed by Bauer et al. [1]. The study on the bondage number was motivated by the increasing importance in the design and analysis of interconnection networks. Since then, the bondage number has attracted much attention of the researchers. Recently, several authors have focused on bondage number, obtained lots of results and also left many conjectures and open problems. In this paper we establish domination number and bondage number for some families of graphs.

We use the following definitions in the subsequent sequel.

Definition 1.1. [2] The cocktail party graph of order $n$, (also called the hyperoctahedral graph) is the graph consisting of two rows of paired nodes in which all nodes but the paired ones are connected with a graph edge.

Definition 1.2. The coxeter graph is a nonhamiltonian cubic symmetric graph on 28 vertices and 42 edges.

Definition 1.3. The crown graph for an integer $n \geq 3$ is the graph with vertex set $\left\{\left(x_{i}, x_{j}\right) ; 0 \leq i, j \leq-1, i \neq j\right\}$.

Definition 1.4. [3] A cubic symmetric graph is a symmetric cubic. Since cubic graphs must have an even number of vertices.

Definition 1.5. The doyle graph also known as Holt graph [7], is the quartic symmetric graph on 27 nodes.

Definition 1.6. The folkman graph is a semi symmetric graph that has the minimum possible number of nodes 20 . It has 30 nodes and 45 edges.

Definition 1.7. The levi graph is a generalized polygon which is the point/line incidence graph of the generalized quadrangle.

Definition 1.8. The icosahedral graph has 12 vertices and 30 edges. Since the icosahedral graph is regular and hamiltonian, it has a generalized LCF notation. 


\section{Domination Number for Some Families of Graphs}

In this section, we establish the value of the domination number of cocktail party graph, coxeter graph, crown graph, cubic symmetric graph, doyle graph, folkman graph, levi graph and icosahedral graph.

Lemma 2.1. Let $C_{n}$ be a cocktail party graph. Then $\gamma\left(C_{n}\right)=2$.

Proof. Let $\left\{a_{0}, a_{1}, a_{2}, \ldots, a_{n-1}, b_{0}, b_{1}, b_{2}, \ldots, b_{n-1}\right\}$ be the vertices of $C_{n}$. Let

$A=\left\{a_{0}, a_{1}, a_{2}, \ldots, a_{n-1}\right\}$ and $B=\left\{b_{0}, b_{1}, b_{2}, \ldots, b_{n-1}\right\}$ be the two sets of vertices of $C_{n}$.

To dominate, we need one vertex among $A$ and one more vertex among $B$. Figure 1 shows that the vertices $\left\{a_{0}, b_{0}\right\}$ form a minimum dominating set. Thus, $\gamma\left(C_{n}\right)=2$.

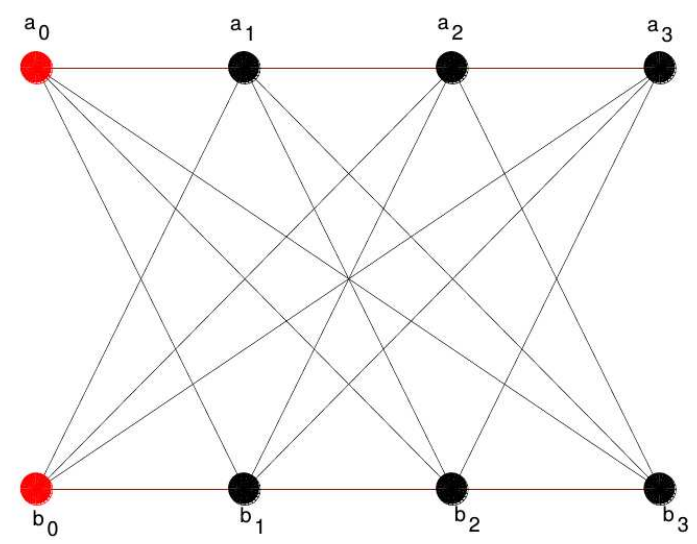

Figure 1: Cocktail party graph $C_{8}$ with $\gamma\left(C_{8}\right)=2$

Lemma 2.2. If $G$ is a coxeter graph, then $\gamma(G)=7$.

Proof. Let $G$ be the coxeter graph with 28 vertices of degree 3. Let $U=$ $\left\{u_{1}, u_{2}, u_{3}, \ldots, u_{7}\right\}$,

$V=\left\{v_{1}, v_{2}, v_{3}, \ldots, v_{7}\right\}$ and $W=\left\{w_{1}, w_{2}, w_{3}, \ldots, w_{14}\right\}$ be the three sets of vertices of $G$. To domination, we need 7 vertices. Figure 2 shows that the vertices $\left\{v_{1}, v_{2}, v_{3}, \ldots, v_{7}\right\}$ form a minimum dominating set. Thus, $\gamma(G)=7$.

Lemma 2.3. Let $S_{n}{ }^{o}$ be a crown graph. Then $\gamma\left(S_{n}{ }^{o}\right)=2$.

Proof. Let $S_{n}{ }^{o}$ be a crown graph with vertex set $\left\{\left(a_{i}, b_{j}\right) ; 0 \leq i, j \leq n-1, i \neq\right.$ $j$ \} of degree $n-1$. To dominate all these vertices, we need 2 vertices. Figure 3 


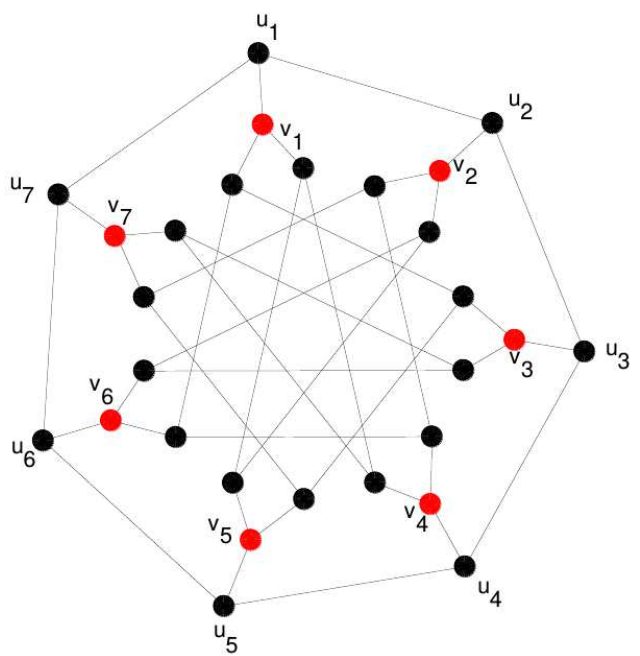

Figure 2: Coxeter graph $G$ with $\gamma(G)=7$

shows that the vertices $\left\{a_{0}, b_{0}\right\}$ form a minimum dominating set of $S_{n}{ }^{o}$. Thus, $\gamma\left(S_{n}{ }^{o}\right)=2$.

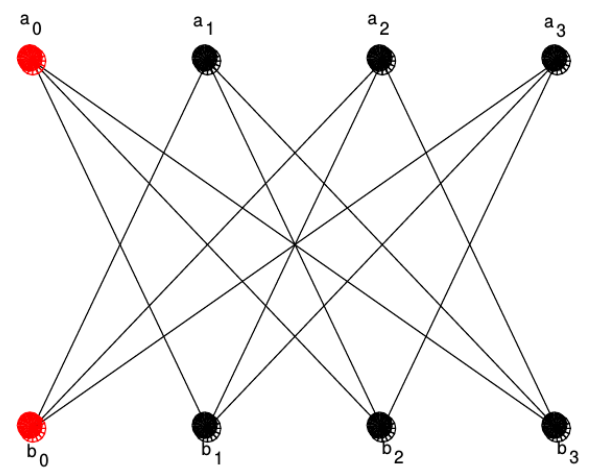

Figure 3: Crown graph $S_{4}{ }^{o}$ with $\gamma\left(S_{4}{ }^{o}\right)=2$

Lemma 2.4. Let $G$ be a cubic symmetric graph. Then $\gamma(G)=2$.

Proof. By definition of cubic symmetric graph, there are 8 vertices with degree 3. To dominate all these vertices, we need 2 vertices. Figure 4 shows that the vertices $\left\{u_{1}, u_{4}\right\}$ form a minimum dominating set of $G$. Thus, $\gamma(G)=2$.

Lemma 2.5. Let $G$ be a doyle graph. Then $\gamma(G)=9$. 


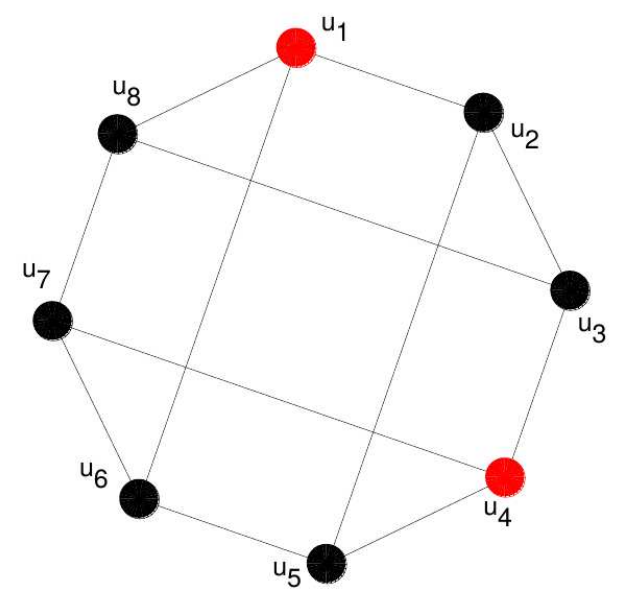

Figure 4: Cubic symmetric graph $G$ with $\gamma(G)=2$

Proof. Let $G$ be the doyle graph with 27 vertices. Let $U=\left\{u_{1}, u_{2}, u_{3}, \ldots, u_{9}\right\}$, $V=\left\{v_{1}, v_{2}, v_{3}, \ldots, v_{9}\right\}$ and $W=\left\{w_{1}, w_{2}, w_{3}, \ldots, w_{9}\right\}$ be the three sets of vertices of $G$ with degree 4 . To domination, we need 9 vertices. Figure 5 shows that the vertices $\left\{u_{1}, u_{2}, u_{3}, \ldots, u_{9}\right\}$ form a minimum dominating set. Thus, $\gamma(G)=9$.

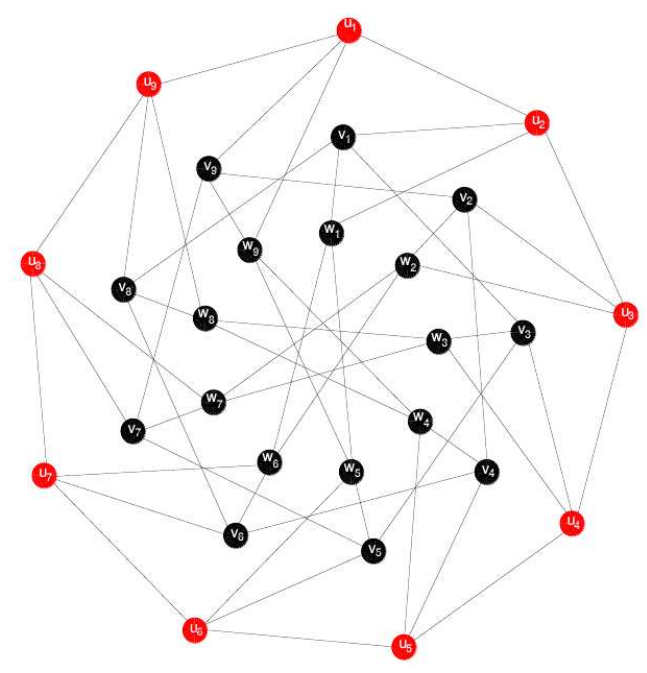

Figure 5: Doyle Party graph $G$ with $\gamma(G)=9$ 
Lemma 2.6. If $G$ is a folkman graph, then $\gamma(G)=6$.

Proof. Let $G$ be the folkman graph with 20 vertices with degree 4 .

Let $V=\left\{a_{0}, a_{1}, a_{2}, a_{3}, a_{4}, b_{0}, b_{1}, b_{2}, b_{3}, b_{4}, c_{0}, c_{1}, c_{2}, c_{3}, c_{4}, d_{0}, d_{1}, d_{2}, d_{3}, d_{4}\right\}$ be the vertices of $G$. To dominate all these vertices, we need 6 vertices. Figure 6 shows that the vertices $\left\{a_{1}, a_{2}, b_{0}, b_{3}, c_{3}, d_{2}\right\}$ form a minimum dominating set of $G$. Thus, $\gamma(G)=6$.

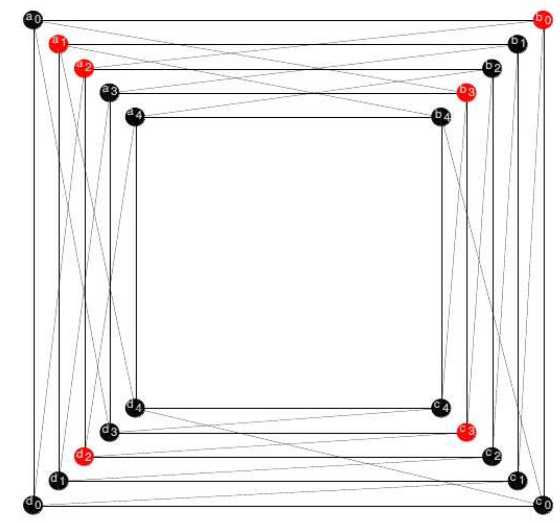

Figure 6: Folkman graph $G$ with $\gamma(G)=6$

Lemma 2.7. Let $G$ be a levi graph. Then $\gamma(G)=10$.

Proof. Let $G$ be the levi graph with 30 vertices. Let $U=\left\{u_{1}, u_{2}, u_{3}, \ldots, u_{10}\right\}$ with degree $4, V=\left\{v_{1}, v_{2}, v_{3}, \ldots, v_{10}\right\}$ with degree 2 and $W=\left\{w_{1}, w_{2}, w_{3}, \ldots\right.$, $\left.w_{10}\right\}$ with degree 4 be the three sets of vertices of $G$. To domination, we need 10 vertices. Figure 7 shows that the vertices $\left\{v_{1}, v_{2}, v_{3}, \ldots, v_{10}\right\}$ form a minimum dominating set. Thus, $\gamma(G)=10$.

Lemma 2.8. Let $G$ be a icosahedral graph. Then $\gamma(G)=2$.

Proof. $G$ be the icosahedral graph with 12 vertices with degree 5 . Let $U=\left\{u_{1}, u_{2}, u_{3}, \ldots, u_{6}\right\}, V=\left\{v_{1}, v_{2}, v_{3}, \ldots, v_{6}\right\}$ be the two sets of vertices of $G$. To domination, we need 2 vertices. Figure 8 shows that the vertices $u_{1}, u_{4}$ form a minimum dominating set. Thus, $\gamma(G)=2$.

\section{Domination Bondage Number for Some Families of Graphs}

In this section, we establish the value of the bondage number of cocktail party graph, coxeter graph, crown graph, cubic symmetric graph, doyle graph, folk- 


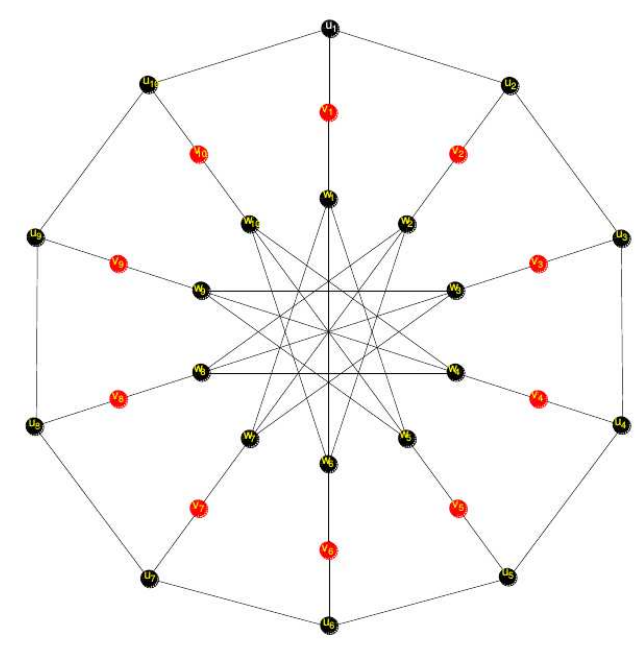

Figure 7: Levi graph $G$ with $\gamma(G)=10$

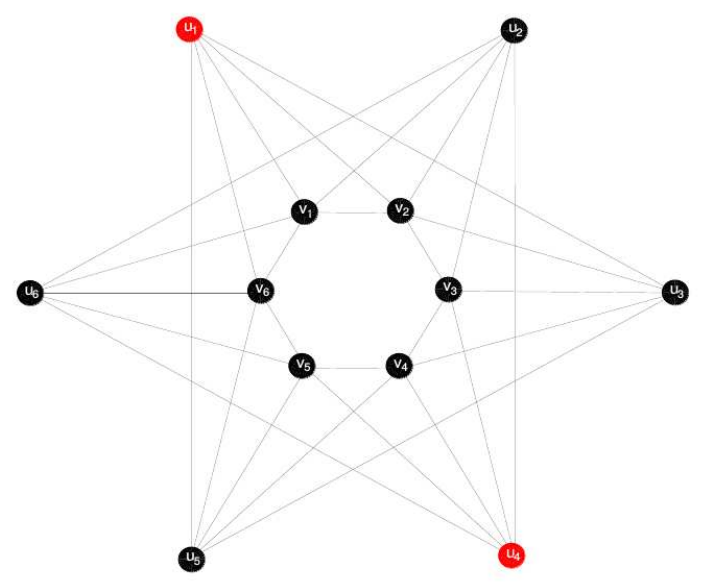

Figure 8: Icosahedral graph $G$ with $\gamma(G)=2$

man graph, levi graph and icosahedral graph.

Theorem 3.1. Let $G$ be a cocktail party graph. Then $B d \gamma(G)=3$.

Proof. Let $S=\left\{a_{0}, b_{0}\right\}$ be a dominating set of cocktail party graph. Let $G^{\prime}$ be obtained from $G$ by removal of an edge $a_{2} a_{3}$. Now there is no change in domination number, $\gamma\left(G^{\prime}\right)=\gamma(G)$. Let $G^{\prime \prime}$ be obtained from $G$ by removal 
of the edges $a_{2} a_{3}$ and $a_{3} b_{1}$. Now there is no change in domination number, $\gamma\left(G^{\prime \prime}\right)=\gamma(G)$. Let $G^{\prime \prime \prime}$ be obtained from $G$ by removal of the edges $a_{2} a_{3}, a_{3} b_{1}$ and $a_{3} b_{0}$. To dominate $a_{3}$, we need one vertex not belongs to $S$. Therefore, $S=\left\{a_{0}, b_{0}, b_{2}\right\}$. Thus, $\gamma\left(G^{\prime \prime \prime}\right)=3$. Figure 15 shows that the domination number of $G^{\prime \prime \prime}$ is greater than the domination number of $G$. This completes the proof.

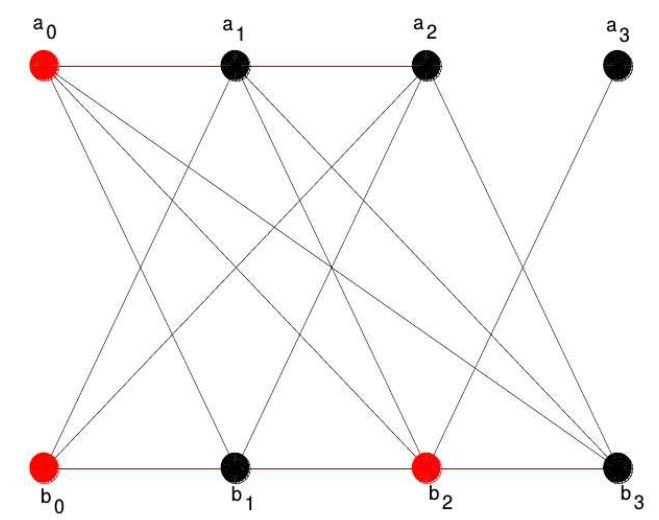

Figure 9: Cocktail Party graph $G^{\prime \prime \prime}$ with $\gamma\left(G^{\prime \prime \prime}\right)=3$

Theorem 3.2. Let $G$ be a coxeter graph. Then $B d \gamma(G)=1$.

Proof. Let $S=\left\{v_{1}, v_{2}, v_{3}, \ldots, v_{7}\right\}$ be a dominating set of coxeter graph. Let $G^{\prime}$ be obtained from $G$ by removal of an edge $u_{1} v_{1}$. To dominate $u_{1}$, we need one vertex not belongs to $S$. Therefore, $S=\left\{u_{1}, v_{1}, v_{2}, v_{3}, \ldots, v_{7}\right\}$. Thus, $\gamma\left(G^{\prime}\right)=8$. Figure 10 shows that the domination number of $G^{\prime}$ is greater than the domination number of $G$. This completes the proof.

Theorem 3.3. If $S_{n}{ }^{o}$ is a crown graph, then $B d \gamma\left(S_{n}{ }^{o}\right)=2$.

Proof. Let $S=\left\{a_{0}, b_{0}\right\}$ be a dominating set of crown graph. Since each vertex having degree $n-1$. Let $\left(S_{n}{ }^{o}\right)^{1}$ be obtained from $S_{n}{ }^{o}$ by removal of an edge $a_{3} b_{1}$. Now there is no change in domination number, $\gamma\left(S_{n}{ }^{o}\right)^{1}=\gamma\left(S_{n}{ }^{o}\right)$. Let $\left(S_{n}{ }^{o}\right)^{2}$ be obtained from $S_{n}{ }^{o}$ by removal of the edges $a_{3} b_{1}$ and $a_{3} b_{0}$. To dominate $a_{3}$, we need one vertex not belongs to $S$. Therefore, $S=\left\{a_{0}, b_{0}, b_{2}\right\}$. Thus, $\gamma\left(S_{n}{ }^{o}\right)^{2}=3$. Figure 11 shows that the domination number of $\left(S_{n}{ }^{o}\right)^{2}$ is greater than the domination number of $S_{n}{ }^{o}$. This completes the proof.

Theorem 3.4. If $G$ is a cubic symmetric graph, then $B d \gamma(G)=2$. 


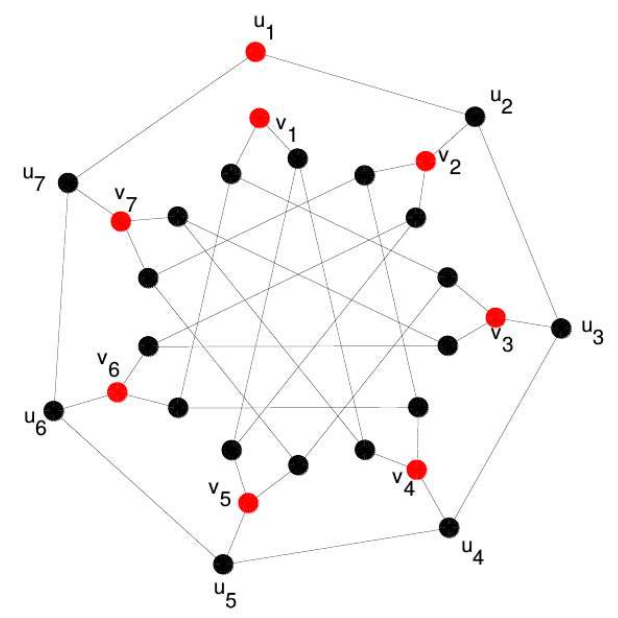

Figure 10: Coxeter graph $G^{\prime}$ with $\gamma\left(G^{\prime}\right)=8$

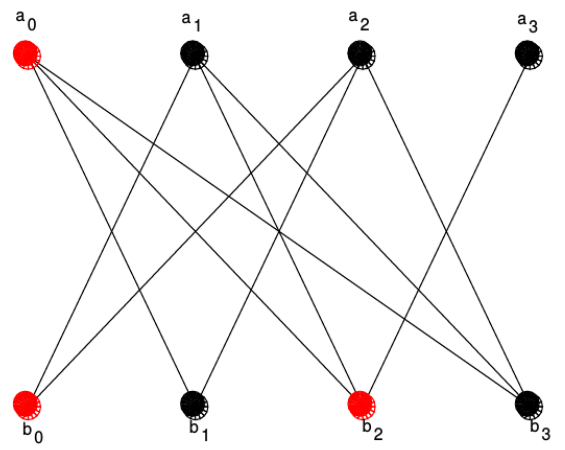

Figure 11: Crown graph $S_{4}{ }^{o}$ with $\gamma\left(S_{n}{ }^{o}\right)^{2}=3$

Proof. Let $S=\left\{u_{1}, u_{4}\right\}$ be a dominating set of cubic symmetric graph. Let $G^{\prime}$ be obtained from $G$ by removal of an edge $u_{2} u_{3}$. Now there is no change in domination number, $\gamma\left(G^{\prime}\right)=\gamma(G)$. Let $G^{\prime \prime}$ be obtained from $G$ by removal of the edges $u_{2} u_{3}$ and $u_{1} u_{2}$. To dominate $u_{2}$, we need one vertex not belongs to $S$. Therefore, $S=\left\{u_{1}, u_{4}, u_{5}\right\}$. Thus, $\gamma\left(G^{\prime \prime}\right)=3$. Figure 12 shows that the domination number of $G^{\prime \prime}$ is greater than the domination number of $G$. This completes the proof.

Theorem 3.5. Let $G$ be a doyle graph. Then $B d \gamma(G)=2$.

Proof. Let $S=\left\{u_{1}, u_{2}, u_{3}, \ldots, u_{9}\right\}$ be a dominating set of doyle graph. Let $G$ be obtained from $G$ by removal of an edge $v_{1} w_{1}$. Now there is no change 


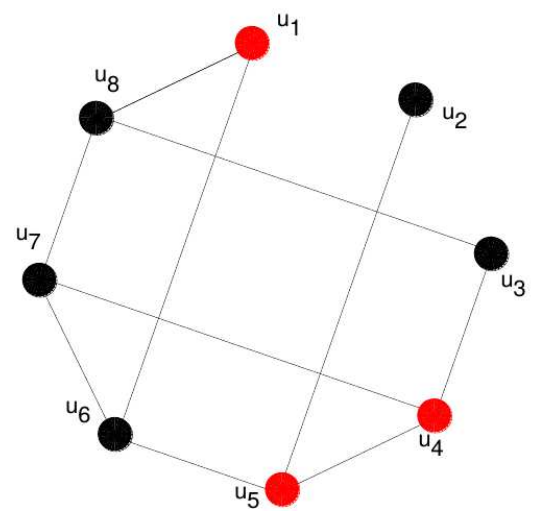

Figure 12: Cubic symmetric graph $G^{\prime \prime}$ with $\gamma\left(G^{\prime \prime}\right)=3$

in domination number, $\gamma\left(G^{\prime}\right)=\gamma(G)$. Let $G^{\prime \prime}$ be obtained from $G$ by removal of the edges $v_{1} w_{1}$ and $v_{1} u_{2}$. To dominate $v_{1}$, we need one vertex. Therefore, $S=\left\{v_{1}, u_{1}, u_{2}, u_{3}, \ldots, u_{9}\right\}$. Thus, $\gamma\left(G^{\prime \prime}\right)=10$. Figure 13 shows that the domination number of $G^{\prime \prime}$ is greater than the domination number of $G$. This completes the proof.

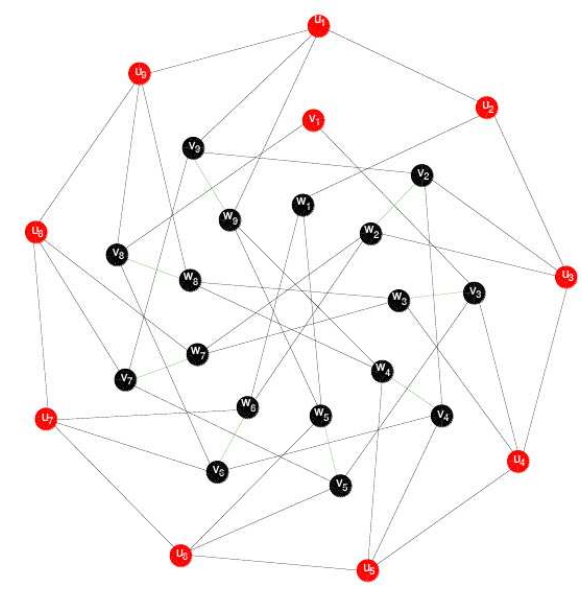

Figure 13: Doyle graph $G^{\prime \prime}$ with $\gamma\left(G^{\prime \prime}\right)=10$

Theorem 3.6. Let $G$ be a folkman graph. Then $B d \gamma(G)=2$.

Proof. Let $S=\left\{a_{1}, a_{2}, b_{0}, b_{3}, c_{3}, d_{2}\right\}$ be a dominating set of folkman graph. Let $G^{\prime}$ be obtained from $G$ by removal of an edge $a_{0} b_{0}$. Now there is no change in domination number, $\gamma\left(G^{\prime}\right)=\gamma(G)$. Let $G^{\prime \prime}$ be obtained from $G$ by removal 
of the edges $a_{0} b_{0}$ and $a_{0} b_{3}$. To dominate $a_{0}$, we need one vertex. Therefore, $S=\left\{a_{0}, a_{1}, a_{2}, b_{0}, b_{3}, c_{3}, d_{2}\right\}$. Thus, $\gamma\left(G^{\prime \prime}\right)=7$. Figure 14 shows that the domination number of $G^{\prime \prime}$ is greater than the domination number of $G$. This completes the proof.

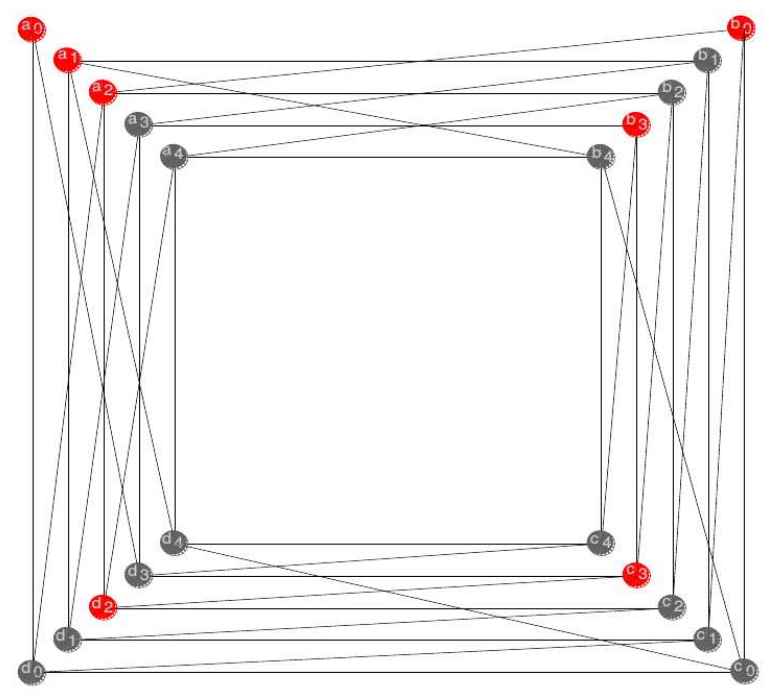

Figure 14: Folkman graph $G^{\prime \prime}$ with $\gamma\left(G^{\prime \prime}\right)=7$

Theorem 3.7. If $G$ is a levi graph, then $B d \gamma(G)=1$.

Proof. Let $S=\left\{v_{1}, v_{2}, v_{3}, \ldots, v_{10}\right\}$ be a dominating set of levi graph. Let $G$ be obtained from $G$ by removal of an edge $u_{1} v_{1}$. To dominate $u_{1}$, we need one vertex. Therefore, $S=\left\{u_{1}, v_{1}, v_{2}, v_{3}, \ldots, v_{10}\right\}$. Thus, $\gamma(G)^{\prime}=11$. Figure 10 shows that the domination number of $G$ is greater than the domination number of $G$. This completes the proof.

Theorem 3.8. If $G$ is a icosahedral graph, then $B d \gamma(G)=4$.

Proof. Let $S=\left\{u_{1}, u_{4}\right\}$ be a dominating set of icosahedral graph. Let $G$ ' be obtained from $G$ by removal of an edge $u_{2} v_{1}$. Now there is no change in domination number, $\gamma\left(G^{\prime}\right)=\gamma(G)$. Let $G^{\prime \prime}$ be obtained from $G$ by removal of the edges $u_{2} v_{1}$ and $u_{2} v_{2}$. Now there is no change in domination number, $\gamma\left(G^{\prime \prime}\right)=\gamma(G)$. Let $G^{\prime \prime \prime}$ be obtained from $G$ by removal of the edges $u_{2} v_{1}, u_{2} v_{2}$ and $u_{2} v_{3}$. Now there is no change in domination number, $\gamma\left(G^{\prime \prime}\right)=\gamma(G)$. Let $G^{\prime \prime \prime \prime}$ be obtained from $G$ by removal of the edges $u_{2} v_{1}, u_{2} v_{2}, u_{2} v_{3}$ and $u_{2} v_{4}$. To dominate $u_{2}$, we need one vertex. Therefore, $S=\left\{u_{2}, u_{1}, u_{4}\right\}$. Thus, $\gamma\left(G^{\prime \prime \prime \prime}\right)=$ 


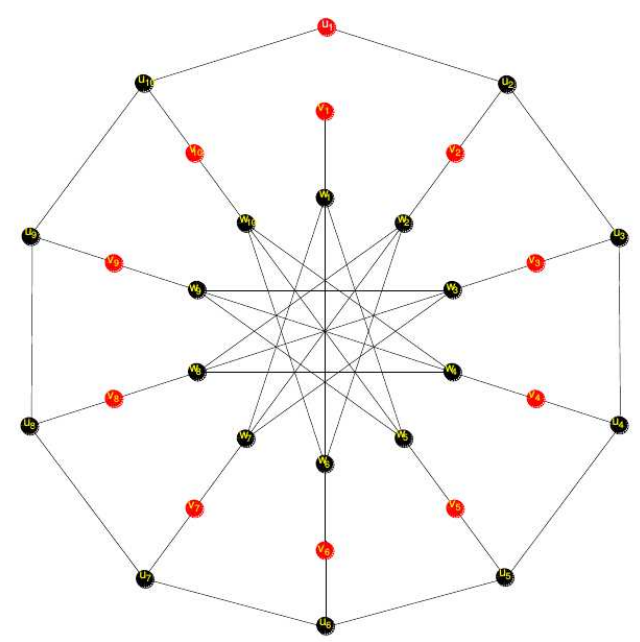

Figure 15: Levi graph $G^{\prime}$ with $\gamma\left(G^{\prime}\right)=11$

3. Figure 16 shows that the domination number of $G^{\prime \prime \prime \prime}$ is greater than the domination number of $G$. This completes the proof.

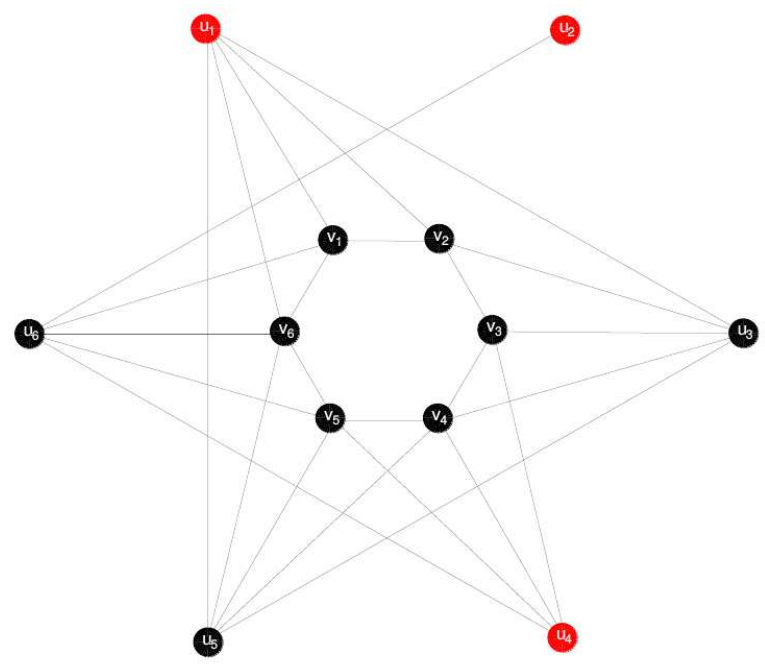

Figure 16: Icosahedral graph $G^{\prime \prime \prime \prime}$ with $\gamma\left(G^{\prime \prime \prime \prime}\right)=3$ 


\section{References}

[1] D. Bauer, F. Harary, J. Nieminen, C.L. Suffel, Domination alteration sets in graphs, Discrete Mathematics, 47 (1983), 153-161.

[2] N.L. Biggs, Algebraic Graph Theory, 2nd ed. Cambridge, England, Cambridge University Press (1993), 17-68.

[3] A.E. Brouwer, A.M. Cohen, A. Neumaier, Distance-Regular Graphs, Springer-Verlag, New York, (1989).

[4] J.F. Fink, M.S. Jacobson, L.F. Kinch, J. Roberts, The bondage number of a graph, Discrete Mathematics, 86 (1990), 47-57.

[5] T.W. Haynes, S.T. Hedetniemi, P.J. Slater, Fundamentals of Domination in Graphs, Marcel Dekker, New York (1997).

[6] T.W. Haynes, S.T. Hedetniemi, P.J. Slater, Domination in Graphs: Advanced Topics, Marcel Dekker, New York (1998).

[7] D.F. Holt, A graph which is edge transitive but not arc transitive, J. Graph Th., 5 (1981), 201-204. 
